\title{
The Infinitesimalization and Reconstruction of Locally Homogeneous Manifolds
}

\author{
Anthony D. BLAOM \\ 22 Ridge Road, Waiheke Island, New Zealand \\ E-mail: anthony.blaom@gmail.com
}

Received May 08, 2013, in final form November 19, 2013; Published online November 26, 2013

http://dx.doi.org/10.3842/SIGMA.2013.074

\begin{abstract}
A linear connection on a Lie algebroid is called a Cartan connection if it is suitably compatible with the Lie algebroid structure. Here we show that a smooth connected manifold $M$ is locally homogeneous - i.e., admits an atlas of charts modeled on some homogeneous space $G / H$ - if and only if there exists a transitive Lie algebroid over $M$ admitting a flat Cartan connection that is 'geometrically closed'. It is shown how the torsion and monodromy of the connection determine the particular form of $G / H$. Under an additional completeness hypothesis, local homogeneity becomes global homogeneity, up to cover.
\end{abstract}

Key words: locally homogeneous; Lie algebroid; Cartan connection; completeness

2010 Mathematics Subject Classification: 53C30; 53C15; 53C07

\section{Introduction}

Let $M$ be a smooth connected manifold. Then $M$ is locally homogeneous if, for some homogeneous space $G / H$, the smooth structure of $M$ can be stiffened to a $\mathcal{G}$-structure, where $\mathcal{G}$ is the pseudogroup of all those local transformations of $G / H$ that are restrictions of a left translation by an element of $G$.

\subsection{Main results}

The chief purpose of this article is to re-examine local homogeneity from the Lie groupoid point of view. This leads, in particular, to the conclusion that a locally homogeneous manifold can be infinitesimalized to obtain a transitive Lie algebroid over $M$, equipped with a flat linear connection $\nabla$ that is compatible with the Lie algebroid structure, i.e. is a flat Cartan connection (see Section 2.1 below). As we shall elucidate, $\nabla$ being flat amounts to the existence of a transitive action by some Lie algebra on $M$ 'twisted' by a monodromy representation.

Not all transitive Lie algebroids equipped with a flat Cartan connection are infinitesimalizations of a locally homogeneous manifold. If $(\mathfrak{g}, \nabla)$ is an infinitesimalization, then $\nabla$ must be geometrically closed, in a sense made precise below. Fortunately, geometric closure is sufficient for reversing the infinitesimalization procedure:

Theorem 1.1. A smooth manifold $M$ is locally homogeneous if and only if there exists a transitive Lie algebroid $\mathfrak{g}$ over $M$ admitting a flat, geometrically closed, Cartan connection $\nabla$.

As we show in Section 5, the particular model $G / H$ that applies is encoded in the torsion and monodromy of $\nabla$. By contrast, in the predominant approach to local homogeneity, one fixes a particular homogeneous space $G / H$ a priori, and asks if $M$ admits $G / H$ as a local model. In practice, this requires one to anticipate an appropriate model, or check several candidates systematically. See, e.g., the survey [10] for this point of view. 


\subsection{Geometric closure}

Let $\mathfrak{g}$ be a transitive Lie algebroid equipped with a flat Cartan connection $\nabla$. Fix an arbitrary point $m_{0} \in M$ and an arbitrary simply-connected open neighbourhood $U$ of $m_{0}$. Let $\mathfrak{g}_{0}$ be the finite-dimensional vector space of $\nabla$-parallel sections over $U$. Because $\nabla$ is flat, $\mathfrak{g}_{0}$ is the same for all choices of $U$, up to obvious identifications. Because $\nabla$ is Cartan, $\mathfrak{g}_{0} \subset \Gamma\left(\mathfrak{g}_{U}\right)$ is a subalgebra (with bracket encoded in the torsion of $\nabla$; see Section 2.5).

Denote the simply-connected Lie group having $\mathfrak{g}_{0}$ as its Lie algebra by $G_{0}$, and let $\mathfrak{h}_{0}$ denote the kernel of the map

$$
\xi \mapsto \# \xi\left(m_{0}\right): \mathfrak{g}_{0} \rightarrow T_{m_{0}} M
$$

Here \#: $\mathfrak{g} \rightarrow T M$ denotes the anchor of $\mathfrak{g}$. It is easy to see that $\mathfrak{h}_{0} \subset \mathfrak{g}_{0}$ is a subalgebra, and we say $\nabla$ is geometrically closed if the connected subgroup $H_{0} \subset G_{0}$ with Lie algebra $\mathfrak{h}_{0}$ is closed in $G_{0}$. In making this definition, the choice of fixed point $m_{0} \in M$ is immaterial, as we will establish later in Section 4.1.

The basic prototype of a Lie algebroid supporting a flat Cartan connection is the action algebroid $\mathfrak{g}_{0} \times M$ associated with some infinitesimal action of a Lie algebra $\mathfrak{g}_{0}$ on $M$ (see Section 2.3). Indeed, locally this is the only example (see Section 2.5). In this case, $\mathfrak{h}_{0} \subset \mathfrak{g}_{0}$ is the isotropy subalgebra at $m_{0}$.

For an example a Lie algebra action that is not geometrically closed, see [12, Example 8] and [13].

\subsection{Discussion}

The novelty of Theorem 1.1 lies mainly in the point of view, as explained in Section 1.1 above. The implications of this change in viewpoint, as it applies to other parts of Cartan's generalization of the Klein Erlangen program, are explored in [4]. In its contemporary conception, this program is described in, e.g., [16].

A Lie algebroid over $M$ equipped with a flat Cartan connection amounts to a Lie algebra action on the universal cover $\tilde{M}$ that suitably respects covering transformations. We reconstruct a locally homogeneous structure on $M$ by applying Cartan's development technique to such actions. Excellent expositions of this technique may be found elsewhere; see, e.g., [16]. That said, Dazord's integrability result [8], and Lie groupoid formalism, provide for an economic treatment of development, which is offered in Section 4. For a detailed treatment of infinitesimal actions of Lie algebras, we refer the reader to $[2,12]$.

It should be noted that the Lie group $G$ occurring in Theorem 1.1 (and in Theorem 1.2 mentioned below) need not be connected. Actually, one may insist that $G$ be connected (indeed simply-connected) but only at the cost of allowing transition functions more general than left translations: rather they may be arbitrary affine transformations (for a definition, see Section 2.4). Our proof of Theorem 1.1 begins with the proof of a variant along these lines.

Theorem 1.1 suggests a two-step strategy for establishing the local homogeneity of a smooth manifold: (i) construct a transitive Lie algebroid $\mathfrak{g}$ over $M$, equipped with some Cartan connection $\nabla$ (not necessarily flat); and (ii) attempt to deform $\nabla$, within the class of Cartan connections, to one that is simultaneously flat and geometrically closed.

For example, associated with any Riemann surface $M$ is a canonical Lie algebroid $\mathfrak{g} \subset J^{1}(T M)$ (the 'isotropy' of the complex structure; see [4]). Associated with any compatible metric $\sigma$ is a subalgebroid $\mathfrak{g}_{\sigma} \subset \mathfrak{g}$. Applying Cartan's method of equivalence, as we have described in [4], one constructs a Cartan connection $\nabla$ on $\mathfrak{g}_{\sigma}$, which extends rather naturally to one on $\mathfrak{g}$. Applying step (ii) above to $\nabla$, one can establish the existence of an atlas of affine coordinates when $M$ has vanishing Euler characteristic $\chi$; applying (ii) to a 'prolongation' $\nabla^{(1)}$ of $\nabla$ - a connection on 
the prolongation $\mathfrak{g}^{(1)} \subset J^{2}(T M)$ of $\mathfrak{g}$ - one obtains an atlas of complex projective coordinates, provided $\chi>1$. That is, we recover a weak version of the uniformization theorem, as in [11]. Details will appear elsewhere.

In any case, Theorem 1.1 furnishes a strategy for constructing topological invariants of smooth manifolds, arising as obstructions to the existence of locally homogeneous structures. Theorem 1.1 also engenders the following question: What are the global analogues of intransitive Lie algebroids over $M$ equipped with flat Cartan connections? The answer: A class of differentiable pseudogroups of transformations on $M$, generalizing the canonical pseudogroups of transformations associated with locally homogeneous structures. As it turns out, such pseudogroups are not necessarily Lie pseudogroups in the classical sense. They nevertheless have a very satisfactory 'Lie theory' which is sketched in [4, Appendix A] and described further in [5].

\subsection{Completeness and homogeneity up to cover}

Suppose there exists a Lie group $G$ acting transitively on the universal cover $\tilde{M}$ of $M$, with $G$ understood to contain the group $\Gamma \cong \pi_{1}(M)$ of covering transformations as a subgroup, and with the action of $G$ on $\tilde{M}$ extending the tautological action of $\Gamma$. In this case $M$ is a double quotient of groups, $M \cong(G / H) / \Gamma$, and $M$ may be said to be homogeneous up to cover. Under a suitable completeness hypothesis, locally homogeneous manifolds are already homogeneous up to cover. See, for example, Thurston's lucid account [17].

To formulate a notion of completeness for Cartan connections leading to a strengthening of Theorem 1.1, let $\mathfrak{g}$ be any Lie algebroid over $M$ and let $t \mapsto X_{t} \in \mathfrak{g}$ be a smooth path, defined on some interval of the real line. Let $m_{t} \in M$ be the footprint of $X_{t}$ and $\dot{m}_{t} \in T M$ its velocity. Then $t \mapsto X_{t}$ is called a $\mathfrak{g}$-path if $\#\left(X_{t}\right)=\dot{m}_{t}$, for all $t$. If $\nabla$ is a linear connection on $\mathfrak{g}$ (not necessarily flat or Cartan), then a $\mathfrak{g}$-path $X_{t} \in \mathfrak{g}$ is called a geodesic of $\nabla$ if $\nabla_{\dot{m}_{t}} X_{t}=0$ for all $t$. A geodesic of the Levi-Cevita connection associated with a Riemannian metric is then a geodesic in the standard sense if we take $\mathfrak{g}=T M$.

The usual argument for the existence of geodesics in Riemannian geometry carries over to the general case: Through every point of $\mathfrak{g}$ there passes a unique geodesic of $\nabla$. We call $\nabla$ complete if every geodesic $X_{t}$ of $\nabla$ can be defined for all time $t \in \mathbb{R}$.

The reader is to be warned that compactness of $M$ is not sufficient for completeness of $\nabla$; a simple counterexample is given in Section 6.1. If, in addition, the image of the monodromy representation has compact closure, then $\nabla$ is indeed complete. Alternatively, if $M$ admits a complete Riemannian metric invariant with respect to a natural representation of the Lie algebroid $\mathfrak{g}$ on $S^{2}\left(T^{*} M\right)$, then $\nabla$ is again complete. Precise statements and proofs are given in Section 6 , along with a proof of the following variation of Theorem 1.1:

Theorem 1.2. A smooth connected manifold $M$ is homogeneous up to cover if and only if there exists a transitive Lie algebroid $\mathfrak{g}$ over $M$ admitting a flat, complete, Cartan connection $\nabla$.

\subsection{Paper outline}

The present article is organized as follows: In Section 2 we review the notion of Cartan connections on Lie algebroids and explain the sense in which flat Cartan connections amount to Lie algebra actions twisted by a monodromy representation (Theorem 2.7). In Section 3, we construct the infinitesimalization $(\mathfrak{g}, \nabla)$ of a locally homogeneous structure on $M$ and observe that it is geometrically closed. This establishes the necessity of the conditions in Theorem 1.1.

In Section 4 we define the development of the infinitesimal action of a Lie algebra and describe its behavior under 'equivariant coordinate changes'. This is applied in Section 5 to reconstruct a locally homogeneous structure from any transitive Lie algebroid equipped with a flat, geometrically closed, Cartan connection, which completes the proof of Theorem 1.1. 
In Section 5 we also explain how the torsion and monodromy of $\nabla$ determine the particular homogeneous model $G / H$ that applies.

In Section 6, we prove Theorem 1.2 and offer some sufficient conditions for completeness. The last section, Section 7, illustrates our results by characterising complete local Lie groups, and by recovering a variant of the well-known classification theorem for complete Riemann manifolds.

\subsection{Notation}

Throughout this paper $\mathfrak{g}$ denotes a Lie algebroid, $\mathfrak{g}_{0}$ and $\mathfrak{h}_{0}$ Lie algebras, $G_{0}, H_{0}, G$ and $H$ Lie groups, and $\mathcal{G}$ a Lie groupoid.

\section{Cartan connections on Lie algebroids}

We assume the reader is familiar with the notion of $\mathfrak{g}$-connections and $\mathfrak{g}$-representations ( $\mathfrak{g}$ a Lie algebroid). See, for example, [3] or [4]. With the exception of Section 2.6, the present section is mostly a summary of [3].

\subsection{Cartan connections defined}

Let $\mathfrak{g}$ be a vector bundle over $M$. Then there is a one-to-one correspondence between linear connections $\nabla$ on $\mathfrak{g}$ and splittings $s_{\nabla}: \mathfrak{g} \rightarrow J^{1} \mathfrak{g}$ of the associated exact sequence

$$
0 \rightarrow T^{*} M \otimes \mathfrak{g} \hookrightarrow J^{1} \mathfrak{g} \rightarrow \mathfrak{g} \rightarrow 0 ;
$$

this correspondence is given by

$$
s_{\nabla} X=J^{1} X+\nabla X \text {. }
$$

Here $J^{1} \mathfrak{g}$ is the vector bundle of one-jets of sections of $\mathfrak{g}$, and the inclusion $T^{*} M \otimes \mathfrak{g} \hookrightarrow J^{1} \mathfrak{g}$ is the morphism whose corresponding map on section spaces sends $d f \otimes X$ to $f J^{1} X-J^{1}(f X)$.

Now suppose that $\mathfrak{g}$ is not just a vector bundle but a Lie algebroid. Then $\nabla$ is a Cartan connection if the vector bundle morphism $s_{\nabla}: \mathfrak{g} \rightarrow J^{1} \mathfrak{g}$ is a morphism of Lie algebroids.

Recall here that $J^{1} \mathfrak{g}$ has a natural Lie algebroid structure determined by the requirement

$$
J^{1}[X, Y]=\left[J^{1} X, J^{1} Y\right], \quad \# J^{1} X=\# X, \quad X, Y \in \Gamma(\mathfrak{g}) .
$$

Here and throughout, the anchor of a Lie algebroid is denoted \#. For details and an explicit formula for the bracket on $J^{1} \mathfrak{g}$, see [3], where it is also shown that $\nabla$ is Cartan if and only if its cocurvature vanishes. The latter is a tensor cocurv $\nabla \in \Gamma\left(\wedge^{2}\left(\mathfrak{g}^{*}\right) \otimes T^{*} M \otimes \mathfrak{g}\right)$ defined by

$$
\operatorname{cocurv} \nabla(X, Y) V=\nabla_{V}[X, Y]-\left[\nabla_{V} X, Y\right]-\left[X, \nabla_{V} Y\right]+\nabla_{\bar{\nabla}_{X} V} Y-\nabla_{\bar{\nabla}_{Y} V} X
$$

In the above formula $\bar{\nabla}$ denotes the so-called associated $\mathfrak{g}$-connection on $T M$, defined by $\bar{\nabla}_{X} V=\# \nabla_{V} X+[\# X, V]$. There is also an associated $\mathfrak{g}$-connection on $\mathfrak{g}$ itself, also denoted $\bar{\nabla}$, and defined by $\bar{\nabla}_{X} Y=\nabla_{Y} X+[X, Y]$. For this connection one can define torsion in the usual way, by

$$
\text { tor } \bar{\nabla}(X, Y)=\bar{\nabla}_{X} Y-\bar{\nabla}_{Y} X-[X, Y]=\nabla_{\# Y} X-\nabla_{\# X} Y+[X, Y] .
$$

When $\nabla$ is Cartan both associated connections are flat, i.e., define representations of the Lie algebroid $\mathfrak{g}$ on $T M$ and $\mathfrak{g}$. 


\subsection{Cartan connection-preserving morphisms}

From a well-known characterization of Lie algebroid morphisms given in, e.g., [14, Proposition 4.3.12], one readily establishes the following:

Proposition 2.1. Let $\mathfrak{g}_{1}$ be a Lie algebroid over $M_{1}$ with Cartan connection $\nabla^{1}$ and $\mathfrak{g}_{2}$ a Lie algebroid over $M_{2}$ with Cartan connection $\nabla^{2}$. Then a connection-preserving vector bundle map $\Phi: \mathfrak{g}_{1} \rightarrow \mathfrak{g}_{2}$, covering some smooth map $\phi: M_{1} \rightarrow M_{2}$, is a Lie algebroid morphism if and only if:

(1) $\# \circ \Phi=T \phi \circ \#$, i.e., $\Phi$ respects anchors; and

(2) $\Phi$ tor $\bar{\nabla}^{1}(X, Y)=\operatorname{tor} \bar{\nabla}^{2}(\Phi X, \Phi Y)$, for all $X, Y \in \mathfrak{g}_{1}$.

Here $\bar{\nabla}^{i}$ is the associated $\mathfrak{g}_{i}$-connection on $\mathfrak{g}_{i}$ (see Section 2.1).

\subsection{Equivariance with twist}

Let $\mathfrak{g}_{0}$ be a finite-dimensional Lie algebra. If $\mathfrak{g}_{0}$ acts smoothly on $M$ from the left $^{1}$ then we denote the corresponding Lie algebra homomorphism $\mathfrak{g}_{0} \rightarrow \Gamma(T M)$ by $\xi \mapsto \xi^{\dagger}$. The canonical flat connection $\nabla$ on the action algebroid $\mathfrak{g}=\mathfrak{g}_{0} \times M$ is an example of a Cartan connection. As we recall in Section 2.5 below, this is, locally, the only example of a flat Cartan connection.

Recall that the anchor of an action algebroid $\mathfrak{g}_{0} \times M$ is defined by $\#(\xi, m)=\xi^{\dagger}(m)$, and that the bracket on sections of $\mathfrak{g}_{0} \times M\left(\mathfrak{g}_{0}\right.$-valued functions on $\left.M\right)$ is given by

$$
[X, Y]:=\nabla_{\# X} Y-\nabla_{\# Y} X+\tau(X, Y),
$$

where $\tau$ is the section of $\wedge^{2}\left(\mathfrak{g}^{*}\right) \otimes \mathfrak{g}$ defined by

$$
\tau(X, Y)(m):=[X(m), Y(m)]_{\mathfrak{g}_{0}}, \quad X, Y \in \Gamma\left(\mathfrak{g}_{0} \times M\right) .
$$

Note that the associated $\mathfrak{g}$-connection $\bar{\nabla}$ on $\mathfrak{g}$ has torsion $\tau$.

Now suppose that $\mathfrak{g}_{0}$ acts smoothly on two manifolds $M_{1}$ and $M_{2}$, and let $\operatorname{End}\left(\mathfrak{g}_{0}\right)$ denote the vector space of Lie algebra endomorphisms of $\mathfrak{g}_{0}$. Then we will say that a smooth map $\phi: M_{1} \rightarrow M_{2}$ is $\mathfrak{g}_{0}$-equivariant with twist $\mu \in \operatorname{End}\left(\mathfrak{g}_{0}\right)$ if $\xi^{\dagger}$ and $(\mu \xi)^{\dagger}$ are $\phi$-related, for all $\xi \in \mathfrak{g}_{0}$. The twist need not be unique. Applying Proposition 2.1, we obtain:

Proposition 2.2. Every connection-preserving vector bundle morphism

$$
\mathfrak{g}_{0} \times M_{1} \stackrel{\Phi}{\rightarrow} \mathfrak{g}_{0} \times M_{2}
$$

is a Lie algebroid morphism if and only if it is of the form $\Phi=\mu \times \phi$, for some smooth $\mathfrak{g}_{0}$ equivariant map $\phi: M_{1} \rightarrow M_{2}$ with twist $\mu \in \operatorname{End}\left(\mathfrak{g}_{0}\right)$. Here $(\mu \times \phi)(\xi, m):=(\mu \xi, \phi(m))$.

The significance of $\mathfrak{g}_{0}$-equivariance in the present context is established in Theorem 2.7 below.

\subsection{Affine transformations}

Let $\mathfrak{g}_{0}$ act on $M$ as above, and let $G_{0}$ be the simply-connected Lie group integrating $\mathfrak{g}_{0}$. As we show in Section 4.3, if the action of $\mathfrak{g}_{0}$ is transitive and geometrically closed, then a $\mathfrak{g}_{0}$-equivariant map $\phi: M \rightarrow M$ with twist $\mu$ can be 'developed' to a map $\phi_{G_{0} / H_{0}}: G_{0} / H_{0} \rightarrow G_{0} / H_{0}$ on a space of left cosets $G_{0} / H_{0}$. Moreover, $\phi_{G_{0} / H_{0}}$ is $G_{0}$-equivariant with twist in an appropriate sense. We pause now to define and characterise such maps.

\footnotetext{
${ }^{1}$ Our convention for defining the bracket on the Lie algebra/algebroid of a Lie group/groupoid is to use right-invariant vector fields.
} 
For every Lie group $G_{0}$ one has the group $\operatorname{Aff}\left(G_{0}\right) \subset \operatorname{Diff}(M)$ of affine transformations, generated by left translations, right translations, and group automorphisms. (Affine transformations of the Abelian group $\mathbb{R}^{n}$ are then affine transformations of $\mathbb{R}^{n}$ in the standard sense of the term.) If $H_{0} \subset G_{0}$ is a subgroup, then some elements of $\operatorname{Aff}\left(G_{0}\right)$ descend to bijections of $G_{0} / H_{0}$ that we also refer to as affine transformations, forming a group denoted by $\operatorname{Aff}\left(G_{0} / H_{0}\right)$. All left translations in $G_{0}$ descend to elements of $\operatorname{Aff}\left(G_{0} / H_{0}\right)$; a right translation by $k \in G_{0}$ descends if and only if $k$ is in the normaliser of $H_{0}$; more generally, an element of $\operatorname{Aff}\left(G_{0} / H_{0}\right)$ is of the form $g \mapsto k \Psi(g)$ for some $k \in G_{0}$ and $\Psi \in \operatorname{Aut}\left(G_{0}\right)$, and descends to an element of $\operatorname{Aut}\left(G_{0} / H_{0}\right)$ if and only if $\Psi\left(H_{0}\right) \subset H_{0}$. For example, $\operatorname{Aff}(\mathrm{SO}(3) / \mathrm{SO}(2)) \cong \mathrm{O}(3)$.

On the other hand, we say that a smooth map $\phi: G_{0} / H_{0} \rightarrow G_{0} / H_{0}$ is $G_{0}$-equivariant with twist $\Psi$ if $\Psi: G_{0} \rightarrow G_{0}$ is a group homomorphism and

$$
\phi(g \cdot x)=\Psi(g) \cdot \phi(x), \quad g \in G_{0}, \quad x \in G_{0} / H_{0} .
$$

The magnanimous reader will readily verify the following characterization:

Proposition 2.3. Let $G_{0}$ and $H_{0} \subset G_{0}$ be connected Lie groups. Then a bijection $\phi: G_{0} / H_{0} \rightarrow$ $G_{0} / H_{0}$ is $G_{0}$-equivariant, with some twist $\Psi \in \operatorname{Aut}\left(G_{0}\right)$, if and only if it is affine.

\subsection{The local form of a Lie algebroid with flat Cartan connection}

For the moment, suppose that $\mathfrak{g}$ is an arbitrary vector bundle over $M$, equipped with a linear connection $\nabla$. Denote the finite-dimensional subspace of $\nabla$-parallel sections of $\mathfrak{g}$ by $\mathfrak{g}_{0}$. In the special case that $\nabla$ is flat, and $M$ is simply-connected, we obtain a connection-preserving isomorphism

$$
\begin{aligned}
& \mathfrak{g}_{0} \times M \stackrel{\sim}{\rightarrow} \mathfrak{g}, \\
& (\xi, m) \mapsto \xi(m) .
\end{aligned}
$$

Proposition 2.4. Let $\mathfrak{g}$ be a Lie algebroid over a smooth connected manifold $M$ and $\nabla$ a Cartan connection, not necessarily flat. Then:

(1) The subspace $\mathfrak{g}_{0} \subset \Gamma(\mathfrak{g})$ of $\nabla$-parallel sections is a Lie subalgebra.

(2) The bracket on $\mathfrak{g}_{0}$ coincides with the torsion of the associated $\mathfrak{g}$-connection on $\mathfrak{g}$, in the sense that

$$
[\xi, \eta](m)=\operatorname{tor} \bar{\nabla}(\xi(m), \eta(m))
$$

for any $m \in M ; \xi, \eta \in \mathfrak{g}_{0}$. Here $\bar{\nabla}$ denotes the associated $\mathfrak{g}$-connection on $\mathfrak{g}$.

(3) The mapping $(\xi, m) \mapsto \# \xi(m): \mathfrak{g}_{0} \times M \rightarrow T M$ defines a smooth action of the Lie algebra $\mathfrak{g}_{0}$ on $M$, making $\mathfrak{g}_{0} \times M$ into an action algebroid.

(4) If $\nabla$ is flat, and $M$ is simply-connected, then the canonical isomorphism $\mathfrak{g} \cong \mathfrak{g}_{0} \times M$ in (2.2) is an isomorphism of Lie algebroids.

\subsection{Monodromy and the global form}

Again suppose that $\mathfrak{g}$ is an arbitrary vector bundle over $M$. Let $\tilde{\mathfrak{g}}$ denote its pullback to a vector bundle over the universal cover $\tilde{M}$ of $M$. Let $\Gamma$ denote the group of covering transformations of $M$. Then it is an elementary observation that there exists a unique lift of the tautological action of $\Gamma$ on $\tilde{M}$ to an action on $\tilde{\mathfrak{g}}$ satisfying the following requirement: For all $X \in \tilde{\mathfrak{g}}$ and $\phi \in \Gamma, X$ and $\phi \cdot X$ have the same image under the canonical projection $\tilde{\mathfrak{g}} \rightarrow \mathfrak{g}$. Evidently $\Gamma$ 
acts on $\tilde{\mathfrak{g}}$ by vector bundle automorphisms, and we recover the original vector bundle over $M$ as a quotient: $\mathfrak{g}=\tilde{\mathfrak{g}} / \Gamma$.

Now suppose $\mathfrak{g}$ is equipped with a flat linear connection $\nabla$ and let $\tilde{\nabla}$ denote the pullback of $\nabla$ to a connection on $\tilde{\mathfrak{g}}$, also flat. Let $\mathfrak{g}_{0} \subset \Gamma(\tilde{\mathfrak{g}})$ denote the vector space of $\tilde{\nabla}$-parallel sections. Then, as $\tilde{M}$ is simply-connected, we have a canonical isomorphism $\tilde{\mathfrak{g}} \cong \mathfrak{g}_{0} \times \tilde{M}$ (as discussed in Section 2.5 above) and consequently $\mathfrak{g} \cong\left(\mathfrak{g}_{0} \times \tilde{M}\right) / \Gamma$. Since the action of $\Gamma$ on $\tilde{\mathfrak{g}}$ described above automatically preserves $\tilde{\nabla}$, it must be of the form

$$
\phi \cdot(\xi, \tilde{m})=\left(\mu_{\phi} \xi, \phi(\tilde{m})\right)=\left(\mu_{\phi} \times \phi\right)(\xi, \tilde{m}),
$$

for some uniquely determined group homomorphism $\mu \mapsto \mu_{\phi}: \Gamma \rightarrow \operatorname{Aut}\left(\mathfrak{g}_{0}\right)$. This is the monodromy representation associated with the flat connection $\nabla$.

Remark 2.5. As the reader will recall, monodromy has the following alternative interpretation. Let $\tilde{m}_{0} \in \tilde{M}$ be a point covering any fixed point $m_{0} \in M$. Then there is an isomorphism $\left.\mathfrak{g}_{0} \cong \mathfrak{g}\right|_{m_{0}}$ in which each $\xi \in \mathfrak{g}_{0}$ corresponds to the image of $\xi\left(\tilde{m}_{0}\right)$ under the canonical projection $\tilde{\mathfrak{g}} \rightarrow \mathfrak{g}$. Also, $\Gamma$ may be identified with the fundamental group $\pi_{1}\left(M, m_{0}\right)$. Under these identifications $\mu_{\phi}(\xi)$ is the $\nabla$-parallel translate of $\left.\xi \in \mathfrak{g}\right|_{m_{0}}$ along any closed path $\gamma$ in $M$ representing $\phi \in \pi_{1}\left(M, m_{0}\right)$. Or, this parallel translation may be viewed in the following way: each $\left.\xi \in \mathfrak{g}\right|_{m_{0}}$ extends to a locally defined $\nabla$-parallel section $X$ of $\mathfrak{g}$, which can be 'analytically continued' around $\gamma$ and re-evaluated at $m_{0}$ to obtain $\mu_{\phi}(\xi)$.

Proposition 2.6. In the scenario above, suppose that $\mathfrak{g}$ is a Lie algebroid and $\nabla$ a flat Cartan connection on $\mathfrak{g}$, so that $\mathfrak{g}_{0}$ is a Lie algebra acting on $\tilde{M}$ (see Proposition 2.4). Then:

(1) The canonical isomorphism $\mathfrak{g} \cong\left(\mathfrak{g}_{0} \times \tilde{M}\right) / \Gamma$ is an isomorphism of Lie algebroids.

(2) The group of covering transformations $\Gamma$ acts in the monodromy representation $\mu \mapsto \mu_{\phi}$ by Lie algebra automorphisms of $\mathfrak{g}_{0}$.

(3) Each covering transformation $\phi \in \Gamma$ is a $\mathfrak{g}_{0}$-equivariant diffeomorphism with twist $\mu_{\phi} \in$ $\operatorname{Aut}\left(\mathfrak{g}_{0}\right)$.

(4) If we restrict the tensor tor $\bar{\nabla} \in \Gamma\left(\wedge^{2}\left(\mathfrak{g}^{*}\right) \otimes \mathfrak{g}\right)$ to define a bracket on the fibre $\left.\mathfrak{g}\right|_{m_{0}}$, then the isomorphism $\left.\mathfrak{g}_{0} \cong \mathfrak{g}\right|_{m_{0}}$ in Remark 2.5 is an isomorphism of Lie algebras. Here $\bar{\nabla}$ denotes the $\mathfrak{g}$-connection on $\mathfrak{g}$ associated with $\nabla$.

Proof. Evidently, there is a unique Lie algebroid structure on $\tilde{\mathfrak{g}}$ such that the projection $\tilde{\mathfrak{g}} \rightarrow \mathfrak{g}$ is a Lie algebroid morphism. With respect to this structure, the action of $\Gamma$ on $\tilde{\mathfrak{g}}$ is by Lie algebroid isomorphisms. Thus the isomorphism $\mathfrak{g} \cong \tilde{\mathfrak{g}} / \Gamma$ may be regarded as a Lie algebroid isomorphism. (None of these statements depend on the existence of a trivialization $\tilde{\mathfrak{g}} \cong \mathfrak{g}_{0} \times \tilde{M}$.) On the other hand, the isomorphism $\tilde{\mathfrak{g}} \cong \mathfrak{g}_{0} \times \tilde{M}$ determined by the flat connection $\tilde{\nabla}$ is a Lie algebroid isomorphism, by Proposition 2.4(4). So (1) holds.

Conclusions (2) and (3) are consequences of Proposition 2.2, and the fact that $\Gamma$ acts on $\tilde{\mathfrak{g}} \cong \mathfrak{g}_{0} \times \tilde{M}$ by Lie algebroid isomorphisms. Conclusion (4) follows from Proposition 2.4(2) and Proposition 2.1.

The constructions above are reversible. Indeed, let $\mathfrak{g}_{0}$ be an arbitrary Lie algebra acting smoothly on the universal cover $\tilde{M}$ of $M$, and let $\phi \mapsto \mu_{\phi}: \Gamma \rightarrow \operatorname{Aut}\left(\mathfrak{g}_{0}\right)$ be a representation of $\Gamma$ by Lie algebra automorphisms of $\mathfrak{g}_{0}$ satisfying (3) (of which there may be more than one). Then the action of $\Gamma$ on the action algebroid $\tilde{\mathfrak{g}}:=\mathfrak{g}_{0} \times \tilde{M}$ defined by $(2.3)$ is by Lie algebroid automorphisms (by Proposition 2.2), implying that the quotient $\mathfrak{g}:=\tilde{\mathfrak{g}} / \Gamma$ is a Lie algebroid. Moreover, the canonical flat Cartan connection on $\tilde{\mathfrak{g}}=\mathfrak{g}_{0} \times \tilde{M}$ drops to a flat Cartan connection on $\mathfrak{g}$ whose monodromy is precisely $\mu$. This establishes the following: 
Theorem 2.7. Let $\Gamma \cong \pi_{1}(M)$ denote the group of covering transformations of $\tilde{M}$. Then there is a natural one-to-one correspondence between: $(i)$ pairs $(\mathfrak{g}, \nabla)$, where $\mathfrak{g}$ is a Lie algebroid over $M$ and $\nabla$ is a flat Cartan connection; and $(i i)$ pairs $\left(\mathfrak{g}_{0}, \mu\right)$, where $\mathfrak{g}_{0}$ is a finite-dimensional Lie algebra acting smoothly on $\tilde{M}$, and $\mu$ is a representation of $\Gamma$ on $\mathfrak{g}_{0}$ by Lie algebra automorphisms such that each covering transformation $\phi \in \Gamma$ is a $\mathfrak{g}_{0}$-equivariant diffeomorphism with twist $\mu_{\phi} \in \operatorname{Aut}\left(\mathfrak{g}_{0}\right)$.

\section{Infinitesimalization}

In this section we prove necessity of the conditions in Theorem 1.1, i.e., for every locally homogeneous manifold $M$, there exists a transitive Lie algebroid $\mathfrak{g}$ over $M$ supporting a flat, geometrically closed, Cartan connection $\nabla$. We refer to the particular pair $(\mathfrak{g}, \nabla)$ constructed below as the locally homogeneous structure's infinitesimalization.

\subsection{Construction of the infinitesimalization}

Suppose $M$ is a locally homogeneous manifold modeled on $G / H$ and let $\psi_{i}: U_{i} \rightarrow G / H, i \in I$, be an atlas of coordinate charts adapted to the model. This means that for each $i, j \in I$ for which $U_{i} \cap U_{j} \neq \varnothing$, we are given an element $g_{j i} \in G$, such that $\left(\psi_{j} \circ \psi_{i}^{-1}\right)(x)=g_{j i} \cdot x$ for each $x \in \psi_{i}\left(U_{i}\right) \cap \psi_{j}\left(U_{j}\right)$. Moreover, the elements $g_{j i}$ satisfy the cocycle conditions $g_{i i}=\mathrm{id}$, and $g_{i j} g_{j k}=g_{i k}$ for all $i, j, k \in I$.

Now let $\mathfrak{g}_{0}$ denote the Lie algebra of $G$. Infinitesimalizing the action of $G$ on $G / H$, we obtain a Lie algebra homomorphism $\xi \mapsto \xi^{\dagger}: \mathfrak{g}_{0} \rightarrow \Gamma(T(G / H))$, which makes $\mathfrak{g}_{0} \times G / H$ into a transitive action algebroid. In particular, each restriction $\mathfrak{g}_{0} \times \psi_{i}\left(U_{i}\right) \subset \mathfrak{g}_{0} \times G / H$ is a transitive action algebroid over $\psi_{i}\left(U_{i}\right)$. We claim that the Lie algebroids $\mathfrak{g}_{0} \times \psi_{i}\left(U_{i}\right), i \in I$, are local trivializations of a single transitive Lie algebroid $\mathfrak{g}$ over $M$, the canonical flat connections on the $\mathfrak{g}_{0} \times \psi_{i}\left(U_{i}\right)$ representing a flat Cartan connection $\nabla$ on $\mathfrak{g}$.

To see this, define an equivalence relation $\sim$ on the set

$$
\left\{(\xi, x, i) \in \mathfrak{g}_{0} \times G / H \times I \mid x \in \psi_{i}\left(U_{i}\right)\right\}
$$

(the disjoint union of the sets $\left.\mathfrak{g}_{0} \times \psi_{i}\left(U_{i}\right), i \in I\right)$ by declaring $(\xi, x, i) \sim\left(\xi^{\prime}, x^{\prime}, i^{\prime}\right)$ whenever there is an $m \in U_{i} \cap U_{i^{\prime}}$ such that $\psi_{i}(m)=x, \psi_{i^{\prime}}(m)=x^{\prime}$ and $\xi^{\prime}=\operatorname{Ad}_{g_{i^{\prime} i}} \xi$. Then the set $\mathfrak{g}$ of equivalence classes is a smooth vector bundle over $M$, with footprint projection $[\xi, x, i] \mapsto \psi_{i}^{-1}(x)$; here $[\xi, x, i]$ denotes the class with representative $(\xi, x, i)$.

The vector bundle $\mathfrak{g}$ admits local trivializations given by

$$
\begin{aligned}
& \left.\mathfrak{g}\right|_{U_{i}} \stackrel{\Psi_{i}}{\longrightarrow} \mathfrak{g}_{0} \times \psi_{i}\left(U_{i}\right), \\
& {[\xi, x, i] \mapsto(\xi, x),}
\end{aligned}
$$

with transition functions given by

$$
\left(\Psi_{j} \circ \Psi_{i}^{-1}\right)(\xi, x)=\left(\operatorname{Ad}_{g_{j i}} \xi, g_{j i} \cdot x\right) .
$$

In particular, the transition functions preserve the canonical flat connections on the action algebroids $\mathfrak{g}_{0} \times \psi_{i}\left(U_{i}\right)$. It follows that there is a (necessarily flat) connection $\nabla$ on $\mathfrak{g}$ that is locally represented by the canonical flat connection on each $\mathfrak{g}_{0} \times \psi_{i}\left(U_{i}\right)$.

If we write $L_{g}(x):=g \cdot x$, then the pushforward of $\xi^{\dagger}$ by the transformation $L_{g}: G / H \rightarrow G / H$ is $\left(\operatorname{Ad}_{g} \xi\right)^{\dagger}$. Using this fact, one easily sees that there is a well-defined vector bundle epimorphism $\#: \mathfrak{g} \rightarrow T M$, defined locally by

$$
\#\left(\Psi_{i}^{-1}(\xi, x)\right)=T \psi_{i}^{-1} \cdot \xi^{\dagger}(x) .
$$


It remains to define a Lie bracket on sections of $\mathfrak{g}$ for which \# is a compatible anchor. To this end, notice that each local trivialization $\Psi_{i}$ identifies a fibre $\left.\mathfrak{g}\right|_{m}\left(m \in U_{i}\right)$ with $\mathfrak{g}_{0}$. This identification depends on the local trivialization chosen, but only up to adjoint transformations of $\mathfrak{g}_{0}$; it consequently transfers the Lie bracket on $\mathfrak{g}_{0}$ to one on $\left.\mathfrak{g}\right|_{m}$ that is trivialization-independent. We let $\tau \in \wedge^{2}\left(\mathfrak{g}^{*}\right) \otimes \mathfrak{g}$ denote the tensor whose restriction to each fibre $\left.\mathfrak{g}\right|_{m}$ is the Lie bracket just defined. A bracket on $\mathfrak{g}$ is then given by (cf. (2.1)):

$$
[X, Y]:=\nabla_{\# X} Y-\nabla_{\# Y} X+\tau(X, Y) .
$$

With this bracket $\mathfrak{g}$ becomes a transitive Lie algebroid and the local trivializations $\Psi_{i}:\left.\mathfrak{g}\right|_{U_{i}} \rightarrow$ $\mathfrak{g}_{0} \times \psi_{i}\left(U_{i}\right)$ become connection-preserving Lie algebroid morphisms. In particular, the connection $\nabla$ is Cartan and geometrically closed because its local representatives are.

\section{The development of Lie algebra actions}

To reconstruct a locally homogeneous structure, from a pair $(\mathfrak{g}, \nabla)$ satisfying the conditions in Theorem 1.1, we will use Cartan's development technique. We pause here to describe development using the economy afforded by Lie groupoid language, and to show that development is suitably equivariant. Before doing so, we argue that geometric closure, as defined in Section 1.2, is independent of the choice of fixed point $m_{0} \in M$.

\subsection{Geometric closure recharacterized}

Evidently a flat Cartan connection $\nabla$ on a transitive Lie algebroid $\mathfrak{g}$ over $M$ is geometrically closed 'at the point $m_{0} \in M$ ' if and only if $\tilde{\nabla}$ is geometrically closed at some point $\tilde{m}_{0} \in \tilde{M}$ covering $m_{0}$. Here $(\tilde{\mathfrak{g}}, \tilde{\nabla})$ denotes the lift of $(\mathfrak{g}, \nabla)$ to the universal cover $\tilde{M}$, as described in Section 2.6. So, without loss of generality, we now suppose $M$ is simply-connected.

According to Proposition 2.4(4), $\mathfrak{g}$ is isomorphic to an action algebroid $\mathfrak{g}_{0} \times M$, where $\mathfrak{g}_{0}$ has the same meaning as in Section 1.2. According to Dazord [8], all action algebroids are integrable. So there is a Lie groupoid $\mathcal{G}$ integrating $\mathfrak{g}_{0} \times M$, which we may take to be source-simply-connected [7, Lie I].

Fix some $m_{0} \in M$ and let $\mathcal{G}_{m_{0}}^{m_{0}}$ denote the isotropy group at $m_{0}$ (the group of arrows of $\mathcal{G}$ simultaneously beginning and ending at $m_{0}$ ).

Lemma 4.1. The isotropy group $\mathcal{G}_{m_{0}}^{m_{0}}$ is connected.

Proof. Since we assume $\mathfrak{g}_{0}$ acts transitively, $\mathcal{G}$ is a transitive Lie groupoid (because its orbits are disjoint and open and $M$ is connected). Consequently, if $P \subset \mathcal{G}$ denotes the source-fibre over $m_{0}$ (the subset of all arrows in $\mathcal{G}$ beginning at $m_{0}$ ), then $P$ is a principal $\mathcal{G}_{m_{0}}^{m_{0}}$-bundle over $M$, with the Lie group $\mathcal{G}_{m_{0}}^{m_{0}}$ acting on $P$ from the right. The bundle projection $P \rightarrow M$ is just the restriction of the target-projection $\mathcal{G} \rightarrow M$ of the groupoid $\mathcal{G}$. For this principal bundle we have a corresponding long exact sequence in homotopy,

$$
\cdots \rightarrow \pi_{1}\left(\mathcal{G}_{m_{0}}^{m_{0}}\right) \rightarrow \pi_{1}(P) \rightarrow \pi_{1}(M) \rightarrow \pi_{0}\left(\mathcal{G}_{m_{0}}^{m_{0}}\right) \rightarrow \pi_{0}(P) \rightarrow \cdots
$$

Since $P$ is connected and $M$ is simply-connected, $\pi_{0}\left(\mathcal{G}_{m_{0}}^{m_{0}}\right)$ is trivial.

Let $G_{0}$ and $H_{0}$ have the meanings given in Section 1.2. Then then there exists a Lie groupoid morphism $\Omega: \mathcal{G} \rightarrow G_{0}$ integrating the canonical projection $\mathfrak{g}_{0} \times M \rightarrow \mathfrak{g}_{0}$ (a morphism of Lie algebroids) [7, Lie II]. Evidently the subgroups $\Omega\left(\mathcal{G}_{m_{0}}^{m_{0}}\right)$ and $H_{0}$ of $G_{0}$ have the same Lie algebra $\mathfrak{h}_{0}$. By the lemma they must coincide. This proves: 
Proposition 4.2. The Cartan connection $\nabla$ is geometrically closed if and only if the (necessarily connected) Lie group $\Omega\left(\mathcal{G}_{m_{0}}^{m_{0}}\right)$ is closed in $G_{0}$.

The independence of the choice of point $m_{0} \in M$ is now clear: If $m_{0}^{\prime} \in M$ is a second point then, by the transitivity of $\mathcal{G}$, there exits an arrow $p \in \mathcal{G}$ from $m_{0}$ to $m_{0}^{\prime}$, in which case $\mathcal{G}_{m_{0}^{\prime}}^{m_{0}^{\prime}}=p \mathcal{G}_{m_{0}}^{m_{0}} p^{-1}$ and $\Omega\left(\mathcal{G}_{m_{0}^{\prime}}^{m_{0}^{\prime}}\right)=g \Omega\left(\mathcal{G}_{m_{0}}^{m_{0}}\right) g^{-1}$, where $g=\Omega(p)$.

\subsection{Development defined}

Let $\mathfrak{g}_{0}$ be any finite-dimensional Lie algebra acting smoothly on $M$. Assume the action is transitive and geometrically closed. The development is always defined as a map from the universal cover $\tilde{M}$ (on which $\mathfrak{g}_{0}$ acts also) so, without loss of generality, we suppose once more that $M$ is simply-connected. We again denote by $G_{0}$ the simply-connected Lie group having $\mathfrak{g}_{0}$ as Lie algebra, and let $\mathcal{G}, \mathcal{G}_{m_{0}}^{m_{0}}, P$, and $\Omega: \mathcal{G} \rightarrow G_{0}$ have the meanings given in the preceding Section 4.1. By Proposition 4.2 and geometric closure, $H_{0}:=\Omega\left(\mathcal{G}_{m_{0}}^{m_{0}}\right)$ is a closed subgroup of $G_{0}$, so that $G_{0} / H_{0}$ is a smooth Hausdorff manifold.

Because $\Omega: \mathcal{G} \rightarrow G_{0}$ integrates the projection $\mathfrak{g}_{0} \times M \rightarrow \mathfrak{g}_{0}$ (a point-wise isomorphism), its restriction to $P$ is a local diffeomorphism $\Omega: P \rightarrow G_{0}$. Using the fact that $\Omega$ is a groupoid morphism, we see that $\Omega$ sends orbits of $\mathcal{G}_{m_{0}}^{m_{0}}$ in $P$ to orbits of $H_{0}$ in $G_{0}$ (left cosets). Since $P / \mathcal{G}_{m_{0}}^{m_{0}} \cong M$, it follows that $\Omega: P \rightarrow G_{0}$ descends to a map $D: M \rightarrow G_{0} / H_{0}$ :

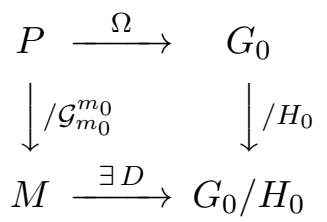

This is the development determined by the arbitrary choice of point $m_{0}$. One has $D(m)=$ $\Omega(p) H_{0}$, where $p \in \mathcal{G}$ is any arrow from $m_{0}$ to $m$.

Proposition 4.3. The development $D: M \rightarrow G_{0} / H_{0}$ is a local diffeomorphism.

Proof. This is a straightforward consequence of the fact that the principal bundle projection $P \rightarrow M$ is a surjective submersion (and so admits local sections), $\Omega: P \rightarrow G_{0}$ is a local diffeomorphism, and $\operatorname{dim}\left(\mathcal{G}_{m_{0}}^{m_{0}}\right)=\operatorname{dim}\left(H_{0}\right)$.

Restricting the development to sufficiently small open sets in $M$, we obtain an atlas of charts trivially adapted the homogeneous space $G_{0} / H_{0}$ :

Corollary 4.4. If a finite-dimensional Lie algebra $\mathfrak{g}_{0}$ acts on a simply-connected manifold $M$, and this action is transitive and geometrically closed, then $M$ is locally homogeneous. Indeed in that case there exists a closed subgroup $H_{0} \subset G_{0}$ of the simply-connected Lie group integrating $\mathfrak{g}_{0}$, and an atlas of charts adapted to the homogeneous model $G_{0} / H_{0}$ whose transition functions are all identity transformations.

\subsection{Behavior under equivariant coordinate changes}

In order to describe how development transforms under equivariant coordinate changes, we wish to associate, with each $\mathfrak{g}_{0}$-equivariant diffeomorphism $\phi: M \rightarrow M$ with twist $\mu \in \operatorname{Aut}\left(\mathfrak{g}_{0}\right)$, a corresponding diffeomorphism

$$
\phi_{G_{0} / H_{0}}: G_{0} / H_{0} \rightarrow G_{0} / H_{0}
$$

To this end, we require the following: 
Lemma 4.5. Let $\phi: M \rightarrow M$ be any smooth $\mathfrak{g}_{0}$-equivariant map with twist $\mu \in \operatorname{End}\left(\mathfrak{g}_{0}\right)$. Let $\hat{\mu}: G_{0} \rightarrow G_{0}$ denote the unique group homomorphism with derivative $\mu$, and let $(\mu \times \phi)^{\wedge}: \mathcal{G} \rightarrow \mathcal{G}$ denote the unique Lie groupoid morphism with derivative $\mu \times \phi: \mathfrak{g}_{0} \times M \rightarrow \mathfrak{g}_{0} \times M$. Then the following diagram commutes:

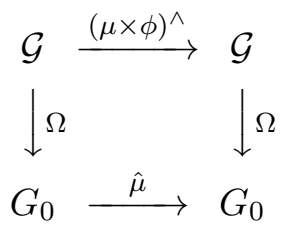

Proof. The Lie groupoid morphisms $\Omega \circ(\mu \times \phi)^{\wedge}$ and $\hat{\mu} \circ \Omega$ have the same derivatives, namely $(\xi, m) \mapsto \mu \xi: \mathfrak{g}_{0} \times M \rightarrow \mathfrak{g}_{0}$. By the uniqueness part of the generalization to Lie groupoids of Lie's second integrability theorem [7, Lie II], these morphisms must coincide.

Lemma 4.6. Suppose $\phi: M \rightarrow M$ is a $\mathfrak{g}_{0}$-equivariant diffeomorphism with twist $\mu \in \operatorname{Aut}\left(\mathfrak{g}_{0}\right)$. Let $q \in \mathcal{G}$ be any arrow from $m_{0}$ to $\phi\left(m_{0}\right)$, and let $\hat{\mu}$ be the unique automorphism of $G_{0}$ with derivative $\mu$. Then $\hat{\mu}\left(H_{0}\right)=\Omega(q) H_{0} \Omega(q)^{-1}$.

Proof. Because, in the notation of Lemma 4.5, the groupoid automorphism $(\phi \times \mu)^{\wedge}: \mathcal{G} \rightarrow \mathcal{G}$ covers $\phi: M \rightarrow M$, and because $q \in \mathcal{G}$ is an arrow from $m_{0}$ to $\phi\left(m_{0}\right)$, we have $(\phi \times \mu)^{\wedge}\left(\mathcal{G}_{m_{0}}^{m_{0}}\right)=$ $q \mathcal{G}_{m_{0}}^{m_{0}} q^{-1}$. Applying that lemma, we compute

$$
\hat{\mu}\left(H_{0}\right)=\hat{\mu}\left(\Omega\left(\mathcal{G}_{m_{0}}^{m_{0}}\right)\right)=\Omega\left((\phi \times \mu)^{\wedge}\left(\mathcal{G}_{m_{0}}^{m_{0}}\right)\right)=\Omega\left(q \mathcal{G}_{m_{0}}^{m_{0}} q^{-1}\right)=\Omega(q) H_{0} \Omega(q)^{-1} .
$$

Lemma 4.6 implies that the map $\phi_{G_{0} / H_{0}}: G_{0} / H_{0} \rightarrow G_{0} / H_{0}$, given implicitly by

$$
\phi_{G_{0} / H_{0}}\left(g H_{0}\right)=\hat{\mu}(g) \Omega(q) H_{0}
$$

is well-defined. It is also independent of the choice of arrow $q \in \mathcal{G}$ from $m_{0}$ to $\phi\left(m_{0}\right)$, because $\Omega(q) H_{0}=D\left(\phi\left(m_{0}\right)\right)$. Note that despite our choice of notation, $\phi_{G_{0} / H_{0}}$ depends not just on $\phi$ but also on the twist $\mu$.

Proposition 4.7. Let $D: M \rightarrow G_{0} / H_{0}$ be the development associated with some finite-dimensional Lie algebra $\mathfrak{g}_{0}$ acting transitively on $M$. Then, for all $\mathfrak{g}_{0}$-equivariant diffeomorphisms $\phi, \phi^{\prime}: M \rightarrow M$ with twists $\mu, \mu^{\prime}$ respectively, one has:

(1) $\phi_{G_{0} / H_{0}} \circ \phi_{G_{0} / H_{0}}^{\prime}=\left(\phi \circ \phi^{\prime}\right)_{G_{0} / H_{0}}$.

(2) $\phi_{G_{0} / H_{0}}: G_{0} / H_{0} \rightarrow G_{0} / H_{0}$ is $G_{0}$-equivariant with twist $\hat{\mu}$, in the sense that

$$
\phi_{G_{0} / H_{0}}(g \cdot x)=\hat{\mu}(g) \cdot \phi_{G_{0} / H_{0}}(x), \quad g \in G_{0}, \quad x \in G_{0} / H_{0} .
$$

(3) The following diagram commutes:

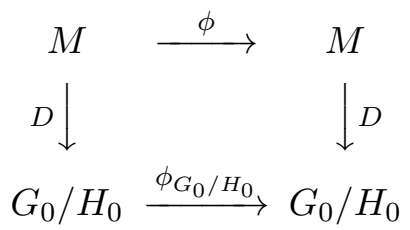

Here $\hat{\mu}$ denotes the unique automorphism of $G_{0}$ integrating the Lie algebra morphism $\mu: \mathfrak{g}_{0} \rightarrow \mathfrak{g}_{0}$.

In other words, if Aut $\left(\mathfrak{g}_{0} \times M\right)$ denotes the group of all $\mathfrak{g}_{0}$-equivariant diffeomorphisms of $M$ with twist, then the map $(\phi, \mu) \mapsto \phi_{G_{0} / H_{0}}$ defines an action of $\operatorname{Aut}\left(\mathfrak{g}_{0} \times M\right)$ on $G_{0} / H_{0}$ by affine transformations (see Proposition 2.3) and with respect to this action and the tautological action of $\operatorname{Aut}\left(\mathfrak{g}_{0} \times M\right)$ on $M$, the development $D: M \rightarrow G_{0} / H_{0}$ is equivariant, in the standard sense of group actions. 
Proof. Let $q, q^{\prime} \in \mathcal{G}$ be arrows from $m_{0}$ to $\phi\left(m_{0}\right), \phi^{\prime}\left(m_{0}\right)$ respectively. Then $q^{\prime \prime}:=(\phi \times \mu)^{\wedge}\left(q^{\prime}\right) q$ is an arrow from $m_{0}$ to $\phi\left(\phi^{\prime}\left(m_{0}\right)\right)$. It follows that for an arbitrary element $g H_{0} \in G_{0} / H_{0}$, we have

$$
\begin{aligned}
\left(\phi \circ \phi^{\prime}\right)_{G_{0} / H_{0}}\left(g H_{0}\right) & =\widehat{\mu \mu^{\prime}}(g) \Omega\left(q^{\prime \prime}\right) H_{0}=\hat{\mu}\left(\widehat{\mu^{\prime}}(g)\right) \Omega\left((\phi \times \mu)^{\wedge}\left(q^{\prime}\right) q\right) H_{0} \\
& =\hat{\mu}\left(\widehat{\mu^{\prime}}(g)\right) \hat{\mu}\left(\Omega\left(q^{\prime}\right)\right) \Omega(q) H_{0}=\hat{\mu}\left(\widehat{\mu^{\prime}}(g) \Omega\left(q^{\prime}\right)\right) \Omega(q) H_{0},
\end{aligned}
$$

where at the beginning of the second line we have applied Lemma 4.5. On the other hand, we have

$$
\phi_{G_{0} / H_{0}}\left(\phi_{G_{0} / H_{0}}^{\prime}\left(g H_{0}\right)\right)=\phi_{G_{0} / H_{0}}\left(\widehat{\mu^{\prime}}(g) \Omega\left(q^{\prime}\right) H_{0}\right)=\hat{\mu}\left(\widehat{\mu^{\prime}}(g) \Omega\left(q^{\prime}\right)\right) \Omega(q) H_{0} .
$$

Comparing this equation with the preceding one establishes (1).

One deduces (2) immediately from the definition of $\phi_{G_{0} / H_{0}}$. Regarding (3), let $m \in M$ be arbitrary and let $p \in \mathcal{G}$ be an arrow from $m_{0}$ to $m$. Then $p^{\prime}:=(\phi \times \mu)^{\wedge}(p) q$ is an arrow from $m_{0}$ to $\phi(m)$. Consequently, we compute

$$
\begin{aligned}
D(\phi(m)) & =\Omega\left(p^{\prime}\right) H_{0}=\Omega\left((\phi \times \mu)^{\wedge}(p)\right) \Omega(q) H_{0} \\
& =\hat{\mu}(\Omega(p)) \Omega(q) H_{0}=\phi_{G_{0} / H_{0}}\left(\Omega(p) H_{0}\right)=\phi_{G_{0} / H_{0}}(D(m)) .
\end{aligned}
$$

At the beginning of the second line we have again applied Lemma 4.5.

\section{Reconstruction}

In this section we complete the proof of Theorem 1.1 by reconstructing a locally homogeneous structure from any transitive Lie algebroid supporting a flat, geometrically closed Cartan connection. We also explain how torsion and monodromy of the connection determine a suitable model $G / H$.

\subsection{The role of torsion and monodromy}

According to the following result, if $M$ satisfies the hypotheses of Theorem 1.1, then it locally homogeneous in the apparently weaker sense of admitting an atlas in which the transition functions are affine transformations of a homogeneous model $G_{0} / H_{0}$ (in the sense of Section 2.4). The advantage of this formulation over the one in Theorem 1.1 is that we may take $G_{0}$ to be simply-connected and $H_{0}$ to be connected.

Proposition 5.1. Let $\mathfrak{g}$ be a transitive Lie algebroid, over a smooth connected manifold $M$, and $\nabla$ a flat Cartan connection on $\mathfrak{g}$. Define a $\mathfrak{g}$-connection $\bar{\nabla}$ on $\mathfrak{g}$ by

$$
\bar{\nabla}_{X} Y=\nabla_{\# Y} X+[X, Y]
$$

and let tor $\bar{\nabla}$ denotes its torsion:

$$
\text { tor } \bar{\nabla}(X, Y)=\bar{\nabla}_{X} Y-\bar{\nabla}_{Y} X-[X, Y]=\nabla_{\# Y} X-\nabla_{\# X} Y+[X, Y] \text {. }
$$

Then, fixing a point $m_{0} \in M$, we have:

(1) The restriction of tor $\bar{\nabla}$ to $\mathfrak{g}_{0}:=\left.\mathfrak{g}\right|_{m_{0}}$ is a Lie bracket on $\mathfrak{g}_{0}$.

Next, let $\Gamma=\pi_{1}\left(M, m_{0}\right)$ denote the fundamental group of $M$ and let $\mu: \Gamma \rightarrow \mathfrak{g}_{0}$ denote the monodromy representation associated with the flat connection $\nabla$. Then: 
(2) $\Gamma$ acts on $\mathfrak{g}_{0}$ by Lie algebra automorphisms. In particular, the monodromy representation integrates to a group homomorphism $\hat{\mu}: \Gamma \rightarrow \operatorname{Aut}\left(G_{0}\right)$, where $G_{0}$ denotes the simplyconnected Lie group with Lie algebra $\mathfrak{g}_{0}$.

Now let $\mathfrak{h}_{0} \subset \mathfrak{g}_{0}=\left.\mathfrak{g}\right|_{m_{0}}$ denote the kernel of the restriction of the anchor $\#: \mathfrak{g} \rightarrow T M$ to $\left.\mathfrak{g}\right|_{m_{0}}$, and let $H_{0} \subset G_{0}$ denote the connected subgroup with Lie algebra $\mathfrak{h}_{0}$. Then:

(3) The connection $\nabla$ is geometrically closed if and only if $H_{0} \subset G_{0}$ is closed, in which case there exists a smooth action

$$
(\phi, x) \mapsto \phi_{G_{0} / H_{0}}(x): \Gamma \times G_{0} / H_{0} \rightarrow G_{0} / H_{0}
$$

of $\Gamma$ on $G_{0} / H_{0}$ by affine transformations, such that $\phi_{G_{0} / H_{0}}$ has twist $\hat{\mu}_{\phi}$.

(4) There is an atlas of charts on $M$ with model $G_{0} / H_{0}$ such that each transition function is a restriction of some $\phi_{G_{0} / H_{0}}, \phi \in \Gamma$.

Remark 5.2. In the proposition statement (but not the subsequent proof) monodromy is to be understood in the usual sense of parallel translation (or analytic continuation) around closed paths; see the Remark 2.5.

Before turning to the proof, let us explain how to recover genuine local homogeneity from the conclusion of the proposition and hence complete the proof of Theorem 1.1. Indeed, let $G=\Gamma \times_{\hat{\mu}} G_{0}$ denote the semidirect product, with multiplication given by

$$
\left(\phi_{1}, g_{1}\right)\left(\phi_{2}, g_{2}\right):=\left(\phi_{1} \phi_{2}, \hat{\mu}_{\phi_{2}}^{-1}\left(g_{1}\right) g_{2}\right) .
$$

Then, as $\phi_{G_{0} / H_{0}}$ is $G_{0}$-equivariant with twist $\hat{\mu}_{\phi}$, the group $G$ acts on $G_{0} / H_{0}$ according to $\left(\phi, g_{0}\right) \cdot x=\phi_{G_{0} / H_{0}}\left(g_{0} \cdot x\right)\left(x \in G_{0} / H_{0}\right)$. As this action is also transitive, we have a canonical isomorphism $G_{0} / H_{0} \cong G / H$, where $H$ is the isotropy at $\operatorname{id} H_{0} \in G_{0} / H_{0}$ of the action by $G$ just defined. Viewing each transition function as a local transformation of $G / H$, it becomes a left-translation by some element of $\Gamma \cong \Gamma \times\{$ id $\} \subset G$.

\subsection{Reconstruction}

To prove the proposition, pull $\mathfrak{g}$ back to a transitive Lie algebroid $\tilde{\mathfrak{g}}$ over the universal cover $\tilde{M}$ of $M$, and $\nabla$ back to a Cartan connection $\tilde{\nabla}$ on $\tilde{\mathfrak{g}}$, as described in Section 2.6. Then, by Proposition 2.4, the finite-dimensional Lie algebra $\mathfrak{g}_{0} \subset \Gamma(\tilde{\mathfrak{g}})$ of $\tilde{\nabla}$-parallel sections acts on $\tilde{M}$ and this action is transitive. That this Lie algebra may be identified with the fibre $\left.\mathfrak{g}\right|_{m_{0}}$, equipped with the bracket described in Proposition 5.1(1), follows from Remark 2.5 and Proposition 2.6(4) (but we make no further use of this interpretation).

Identify $\Gamma$ with the group of covering transformations and let us understand the monodromy representation $\phi \mapsto \mu_{\phi}: \Gamma \rightarrow \operatorname{Aut}\left(\mathfrak{g}_{0}\right)$ in the invariant sense defined in Section 2.6. That Proposition 5.1(2) holds is just Proposition 2.6(2). According to Proposition 2.6(3), each transformation $\phi \in \Gamma$ is a $\mathfrak{g}_{0}$-equivariant diffeomorphism of $M$ with twist $\mu_{\phi}$.

It is clear from Proposition 2.6(4) that geometric closure, in the sense of Section 1.2, is equivalent to the condition in Proposition 5.1(3) above. If $\nabla$ is geometrically closed, we can define the development $D: \tilde{M} \rightarrow G_{0} / H_{0}$ associated with the action of $\mathfrak{g}_{0}$ on $\tilde{M}$, as determined by the choice of some fixed point $\tilde{m}_{0} \in \tilde{M}$ covering $m_{0}$. Defining $\phi_{G_{0} / H_{0}}$ as in (4.1), one obtains an action as described in Proposition 5.1(3) above, on account of Proposition 4.7(1) and (2).

The development $D: \tilde{M} \rightarrow G_{0} / H_{0}$ is a local diffeomorphism (Proposition 4.3) and, according to Proposition 4.7(3), transforms according to

$$
D(\phi(\tilde{m}))=\phi_{G_{0} / H_{0}}(D(\tilde{m})), \quad \phi \in \Gamma, \quad \tilde{m} \in \tilde{M} .
$$


Now cover $M$ with open sets $U_{i}, i \in I$, small enough that each set $U_{i}$ is evenly covered by some open set $\tilde{U}_{i} \subset \tilde{M}$, and such that the restriction of the development $D: \tilde{U}_{i} \rightarrow G_{0} / H_{0}$ is a diffeomorphism onto its image. Refining this covering if necessary, we may arrange that each non-empty intersection $U_{i} \cap U_{j}$ is connected (see, e.g., [6, Theorem I.5.1]). Let $s^{i}: U_{i} \rightarrow \tilde{U}_{i}$ denote the inverse of the restriction $\tilde{U}_{i} \rightarrow U_{i}$ of the covering $\tilde{M} \rightarrow M$. Define charts $\psi^{i}: U_{i} \rightarrow G_{0} / H_{0}$ by $\psi^{i}=D \circ s^{i}$. Then, whenever $U_{i} \cap U_{j} \neq \varnothing$, there exists a covering transformation $\phi^{i j} \in \Gamma$ such that $s^{j}=\phi^{i j} \circ s^{i}$ on $U_{i} \cap U_{j}$. (Here one uses the fact that two local continuous sections of a covering map that have a common connected domain will agree on the entire domain if they agree at one point.) Furthermore, for any $m \in U_{i} \cap U_{j}$, we have, by (5.1),

$$
\psi^{j}(m)=D\left(s^{j}(m)\right)=D\left(\phi^{i j}\left(s^{i}(m)\right)\right)=\phi_{G_{0} / H_{0}}^{i j}\left(D\left(s^{i}(m)\right)\right)=\phi_{G_{0} / H_{0}}^{i j}\left(\psi^{i}(m)\right) .
$$

Whence the maps $\psi^{i}: U_{i} \rightarrow G_{0} / H_{0}, i \in I$, constitute an atlas of charts meeting the requirement in Proposition 5.1(4) above, and this concludes the proof of Proposition 5.1.

\section{Completeness}

\subsection{Completeness in terms of the associated Lie algebra action}

Let $\mathfrak{g}$ be a Lie algebroid on $M$ (not necessarily transitive), let $\nabla$ be a flat Cartan connection on $\mathfrak{g}$, and consider the associated action of the Lie algebra $\mathfrak{g}_{0}$ on $\tilde{M}$ discussed in Section 2.6.

Proposition 6.1. A flat Cartan connection $\nabla$ is complete, in the sense of Section 1.4, if and only if $\mathfrak{g}_{0}$ acts on $\tilde{M}$ by complete vector fields.

Proof. Adopting the notation of Section 2.6, it is easy to see that $\nabla$ is complete if and only if the pullback connection $\tilde{\nabla}$ on $\tilde{\mathfrak{g}}$ is complete. But, according to Proposition 2.4(4), $\tilde{\mathfrak{g}}$ is isomorphic to the action algebroid $\mathfrak{g}_{0} \times \tilde{M}$, the connection $\tilde{\nabla}$ being represented by the canonical flat connection on $\mathfrak{g}_{0} \times \tilde{M}$. But geodesics of the canonical flat connection on $\mathfrak{g}_{0} \times \tilde{M}$ are evidently those paths of the form $X_{t}=\left(\xi, \tilde{m}_{t}\right)$, where $\xi \in \mathfrak{g}_{0}$ and $\tilde{m}_{t}$ is an integral curve of the corresponding vector field $\xi^{\dagger}$.

Compactness of $M$ is insufficient to guarantee completeness:

Counterexample 6.2. Let $M=S^{1}$ be the circle and let $\mathfrak{g}_{0}=\mathbb{R}$ act on $\tilde{M}=\mathbb{R}$ according to $1^{\dagger}=e^{-\theta} \frac{\partial}{\partial \theta}$. Here $\theta$ denotes the standard coordinate function on $\mathbb{R}$. Evidently, $1^{\dagger}$ is not a complete vector field.

Define a representation $\mu: \Gamma \rightarrow \operatorname{Aut}\left(\mathfrak{g}_{0}\right) \cong \mathbb{R} \backslash\{0\}$ of the group of covering transformations $\Gamma \cong \mathbb{Z}$ on $\mathfrak{g}_{0}$ by $\mu_{n}=e^{2 \pi n}$. As a covering transformation, each $n \in \Gamma$ is the map $\theta \mapsto \theta+2 \pi n$, which is a $\mathfrak{g}_{0}$-equivariant map with twist $\mu_{n}$. By the discussion at the end of Section 2.6, the quotient $\mathfrak{g}=\left(\tilde{M} \times \mathfrak{g}_{0}\right) / \Gamma$ is a Lie algebroid over $M=S^{1}$ supporting a flat Cartan connection, but the corresponding Lie algebra action of $\mathfrak{g}_{0}$ on $\tilde{M}$ is, by construction, incomplete.

\subsection{Sufficient conditions for completeness}

Although compactness of $M$ is not sufficient to guarantee completeness, compactness plus simple-connectivity is obviously sufficient, for then $\tilde{M}$ is also compact (and all vector fields on compact manifolds are complete). More generally, we have:

Proposition 6.3. Let $\mathfrak{g}$ be a Lie algebroid over $M$ equipped with a flat Cartan connection $\nabla$, and let $\mu: \Gamma \rightarrow \operatorname{Aut}\left(\mathfrak{g}_{0}\right)$ denote the associated monodromy representation, as described in Section 2.6. Assume that one of the following conditions holds: 
(1) $M$ is compact and the image $\mu(\Gamma) \subset \operatorname{Aut}\left(\mathfrak{g}_{0}\right)$ of the monodromy representation has compact closure, denoted $\bar{\Gamma} \subset \operatorname{Aut}\left(\mathfrak{g}_{0}\right)$; or

(2) $M$ admits a $\mathfrak{g}$-invariant Riemannian metric $\sigma$ and this metric makes $M$ into a complete metric space (automatically true if $M$ is compact).

Then $\nabla$ is complete.

Recall that because we assume $\nabla$ is Cartan, the associated $\mathfrak{g}$-connection $\bar{\nabla}$ on $T M$ defined in Section 2.1 is in fact a $\mathfrak{g}$-representation. There is a corresponding representation of $\mathfrak{g}$ on $S^{2}\left(T^{*} M\right)$, of which $\sigma$ is a section. This explains what is meant by $\mathfrak{g}$-invariance in (2). Note also that $(1)$ is automatic in the special case that $\operatorname{Aut}\left(\mathfrak{g}_{0}\right)$ is already a compact group.

Before proving the preceding proposition, note that by Proposition 6.1 and Theorem 2.7, it suffices to establish the completeness of any action $\xi \mapsto \xi^{\dagger}$ of some Lie algebra $\mathfrak{g}_{0}$ on $\tilde{M}$, under the assumption that each covering transformation $\phi \in \Gamma$ is $\mathfrak{g}_{0}$-equivariant with twist $\mu_{\phi}$. In this case the $\mathfrak{g}$-invariance of the metric $\sigma$ in hypothesis (2) amounts to $\mathfrak{g}_{0}$-invariance of the lifted metric on $\tilde{M}$.

Proof that (1) implies completeness. Fix some $\xi \in \mathfrak{g}_{0}$. We want to show that $\xi^{\dagger}$ is a complete vector field on $\tilde{M}$. We will do this by finding local lower bounds on the time of validity of its flow that are uniform with respect to the action of $\Gamma$; the compactness of $M$ will then imply these bounds are globally uniform. To begin with, let us record that

$$
\phi_{*} \xi^{\dagger}=\left(\mu_{\phi} \xi\right)^{\dagger} \text {, }
$$

for all $\xi \in \mathfrak{g}_{0}$ and $\phi \in \Gamma$; here $\phi_{*}$ denotes pushforward. This is just a restatement of the $\mathfrak{g}_{0}$-equivariance condition on the group of covering transformations.

By the hypothesis (1), there exists a $\Gamma$-invariant inner product on $\mathfrak{g}_{0}$ (take an arbitrary inner product and average with respect to the Haar measure on the compact topological group $\bar{\Gamma}$ ). Equipping $M$ with an arbitrary Riemannian metric, we evidently obtain a $\Gamma$-invariant metric on $\tilde{M}$. At each $\tilde{m} \in \tilde{M}$ we now define

$$
c(\tilde{m}):=\sup _{\xi \neq 0} \frac{\left|\xi^{\dagger}(\tilde{m})\right|}{|\xi|},
$$

where the norms in the numerator and denominator are defined with respect to the metric and inner product just introduced. It is clear $c(\tilde{m})$ is a continuous with respect to $\tilde{m}$.

With the help of (6.1), $\Gamma$-invariance of the metric on $\tilde{M}$, as well as $\Gamma$-invariance of the inner product on $\mathfrak{g}_{0}$, one next shows that $c$ is $\Gamma$-invariant. We can therefore view it as a continuous function $c: M \rightarrow[0, \infty)$, and we have the $\Gamma$-uniform estimate

$$
\left|\xi^{\dagger}(\tilde{m})\right| \leqslant c(\pi(\tilde{m}))|\xi|, \quad \xi \in \mathfrak{g}_{0}, \quad \tilde{m} \in \tilde{M} .
$$

Here $\pi: \tilde{M} \rightarrow M$ denotes the covering projection.

We will now use the following lemma, whose proof is an elementary exercise in Riemannian geometry.

Lemma 6.4. Let $m$ be an arbitrary point of a Riemann manifold $M$, and let $r>0$ be small enough that the open geodesic ball $B_{3 r}(m)$ of radius $3 r$ about $m$ is well-defined ${ }^{2}$. Then for any vector field $V$ on $M$, integral curves of $V$ beginning in $B_{r}(m)$ are well-defined for all times $t \in[-T, T]$, where $T:=r /\|V\|_{m, 2 r}$. Here, $\|V\|_{m, 2 r}$ denotes the supremum of $|V(n)|$ over all $n \in B_{2 r}(m)$.

\footnotetext{
${ }^{2}$ Here we mean a geodesic $=$ normal ball in the sense of, e.g., p. 70 of [9].
} 
So, let $m \in M$ be arbitrary. Then there is $r=r(m)>0$ such that the geodesic ball $B_{3 r}(m) \subset M$ is well-defined, has compact closure, and is evenly covered by a disjoint union of geodesic balls $B_{3 r}(\tilde{m}) \subset \tilde{M}$, one for each $\tilde{m}$ lying over $m$ in the covering. For any such $\tilde{m}$ we have, in the notation of the lemma,

$$
\left\|\xi^{\dagger}\right\|_{\tilde{m}, 2 r} \leqslant C(m)|\xi|, \quad \text { where } \quad C(m):=\sup \left\{c(m) \mid m \in B_{2 r}(m)\right\} .
$$

Here we have applied (6.2) and have $C(m)<\infty$ because $c$ is continuous and $B_{2 r}(m)$ has compact closure. Applying the lemma to the manifold $\tilde{M}$, we conclude that for any initial condition covering a point in $B_{r}(m) \subset M$, the corresponding integral curve of $\xi^{\dagger}$ is defined for all times $t \in[-T(m), T(m)]$, where $T(m):=r(m) /(C(m)|\xi|)>0$. Since $M$ is compact, there is a finite covering $\left\{B_{r\left(m_{j}\right)}\left(m_{j}\right)\right\}_{j=1}^{N}$ of $M$, for some $m_{1}, \ldots, m_{N} \in M$. So an integral curve of $\xi^{\dagger}$ with any initial condition is defined for all times $t \in\left[-T_{\min }, T_{\min }\right]$, where $T_{\min }:=\min _{j=1}^{N} T\left(m_{j}\right)>0$. Since this lower bound on times is independent of the initial condition, the curve is defined for all time.

Proof that (2) implies completeness. In this case every vector field $\xi^{\dagger}$ is a Killing field on the universal cover $\tilde{M}$, to which the metric $\sigma$ lifts. In particular, every integral curve $t \mapsto$ $\tilde{m}(t):(a, b) \rightarrow \tilde{M}$ of $\xi^{\dagger}$ has constant speed. Since $M$ is a complete metric space, so is $\tilde{M}$.

Suppose that $t_{n} \rightarrow b<\infty$, for some sequence $t_{1}, t_{2}, \ldots \in(a, b)$. Because $m(t)$ has constant speed, $m\left(t_{1}\right), m\left(t_{2}\right), \ldots$ is a Cauchy sequence, which must therefore converge to some $m_{b} \in M$. Any integral curve of $\xi^{\dagger}$ beginning at $m_{b}$ may, by uniqueness of integral curves, be regarded as an extension of $m(t)$, i.e., $m(t)$ extends to some time interval $\left(a, b_{+}\right)$with $b_{+}>b$. A similar argument applies to $a$. Whence $\xi^{\dagger}$ is complete by the usual proof by contradiction.

\subsection{The proof of Theorem 1.2}

With Proposition 6.1 in hand, the necessity of completeness in Theorem 1.2 is not difficult to prove, and we now turn to the proof of sufficiency. To that end, we begin with a variant of the result sought in which the model space $G_{0} / H_{0}$ is the quotient of a simply-connected Lie group by a connected subgroup:

Proposition 6.5. Let $\mathfrak{g}$ be a transitive Lie algebroid, over a smooth connected manifold $M$, and $\nabla$ a flat, geometrically closed, complete, Cartan connection on $\mathfrak{g}$. Then:

(1) The subgroup $H_{0} \subset G_{0}$ is closed, when $G_{0}$ and $H_{0}$ have the meanings given in Proposition 5.1, and the universal cover of $M$ is $G_{0} / H_{0}$.

(2) The group $\Gamma \cong \pi_{1}(M)$ of covering transformations is a subgroup of $\operatorname{Aff}\left(G_{0} / H_{0}\right)$, and each element $\phi \in \Gamma$ has twist $\hat{\mu}_{\phi}$. Here $\phi \mapsto \hat{\mu}_{\phi}: \Gamma \rightarrow \operatorname{Aut}\left(G_{0}\right)$ is a group homomorphism determined by the monodromy of $\nabla$ and defined in Proposition 5.1.

To recover Theorem 1.2 from this variant, i.e., to obtain $M \cong(G / H) / \Gamma$, with $\Gamma \subset G$ acting by left translations, one defines $G$ to be the semidirect product $\Gamma \times_{\hat{\mu}} G_{0}$ as described already after the statement of Proposition 5.1.

Proof. Given a pair $(\mathfrak{g}, \nabla)$ satisfying the hypotheses of the proposition, consider the corresponding action of the Lie algebra $\mathfrak{g}_{0}$ on the universal cover $\tilde{M}$ detailed in Section 2.6. In particular, $G_{0}$ is then the simply-connected Lie group integrating $\mathfrak{g}_{0}$. According to Proposition 6.1 and Palais' integrability theorem [15], the action of $\mathfrak{g}_{0}$ can be integrated to an action of the Lie group $G_{0}$. Therefore, in the definition of the development $D: \tilde{M} \rightarrow G_{0} / H_{0}$ of the $\mathfrak{g}_{0}$-action see Section 4.2 , but read $\tilde{M}$ in place of $M$ - we may take $\mathcal{G}$ to be the action groupoid $G_{0} \times \tilde{M}$, and the groupoid morphism $\Omega: \mathcal{G} \rightarrow G_{0}$ is just the projection $\Omega: G_{0} \times \tilde{M} \rightarrow G_{0}$. The subgroup 
$H_{0} \subset G_{0}$ is closed because it is the isotropy subgroup of the $G_{0}$-action at $\tilde{m}_{0}$. Moreover, $P=G_{0} \times\left\{\tilde{m}_{0}\right\}$ and the development $D: \tilde{M} \rightarrow G_{0} / H_{0}$ is seen to be a diffeomorphism. This establishes (1).

In the subsequent identification $\tilde{M} \cong G_{0} / H_{0}$, each covering transformation $\phi \in \Gamma$ and corresponding map $\phi_{G_{0} / H_{0}}$ appearing in Proposition 5.1 is identified. The former therefore have the properties claimed in (2).

\section{Illustrations}

Following are simple illustrations of the theory expounded in the present article.

\subsection{Local Lie groups}

As in [3], we call pair of linear connections $\nabla, \bar{\nabla}$ on a smooth manifold $M$ (more precisely, on its tangent bundle $T M$ ) a dual pair if $\bar{\nabla}_{X} Y-\nabla_{Y} X=[X, Y]$. Notice that a connection $\nabla$ is a Cartan connection precisely when its dual $\bar{\nabla}$ is flat. If both $\nabla$ and $\bar{\nabla}$ are flat, it follows from Theorem 1.1 that $M$ is a locally homogeneous manifold modelled on some Lie group $G$. For the purposes of this section, we accordingly define a local Lie group to be a smooth connected manifold $M$, equipped with a dual pair of simultaneously flat connections $\nabla, \bar{\nabla}$.

A bone fide Lie group $G_{0}$ is a local Lie group in this sense: the canonical flat connections $\nabla, \bar{\nabla}$ - corresponding to right and left trivialization, respectively - furnish the required dual pair. In this case, both connections $\nabla$ and $\bar{\nabla}$ are complete (the geodesics being the right and left cosets of one-parameter subgroups of $G$ ) and have trivial monodromy. Conversely, we have:

Proposition 7.1 (cf. $\S 3.8 .3$ of $[16])$. Let $(M, \nabla, \bar{\nabla})$ be a local Lie group. Then:

(1) The torsion, tor $\bar{\nabla}=-$ tor $\nabla$ of $\bar{\nabla}$, is a $\bar{\nabla}$-parallel tensor whose restriction to any tangent space $\mathfrak{g}_{0}=T_{m_{0}} M, m_{0} \in M$, is a Lie bracket.

If, additionally, $\nabla$ is complete, and $G_{0}$ denotes the simply-connected Lie group integrating $\mathfrak{g}_{0}$, then:

(2) The universal cover of $M$ is $G_{0}$ and the group of covering transformations $\Gamma \cong \pi_{1}(M)$ acts by affine transformations.

If $\nabla$ is both complete and has trivial monodromy, then:

(3) There exists an embedding of $\Gamma$ into $G_{0}$ such that $M \cong G_{0} / \Gamma$.

Proof. The claim (1) is just a special case of Proposition 5.1(1). Conclusion (2) is a special case of Proposition 6.5. Under the additional hypothesis that $\nabla$ has trivial monodromy, Proposition 6.5(2) implies that each element of $\Gamma \subset \operatorname{Aff}\left(G_{0}\right)$ has trivial twist. Each such element is therefore a left translation, allowing us to identify $\Gamma$ with a subgroup of $G_{0}$, as claimed in (3).

Of course, in the case that the embedding $\Gamma \subset G_{0}$ in (3) is normal, $M$ is a global Lie group.

The problem of globalizing a local Lie group structure is also considered in [1] (under the additional assumption that the connection $\bar{\nabla}$ comes from a global parallelism on $M$ ). In particular, it is shown that a necessary condition for globalizability is the vanishing of the class $[w] \in H^{1}(M)$, where $w$ is the closed one-form defined by $w(U)=\operatorname{trace}(\operatorname{tor} \bar{\nabla}(U, \cdot))$, which is closed on account of Bianchi's second identity and the hypothesis curv $\nabla=0$. 


\subsection{Riemannian manifolds with constant scalar curvature}

Let $M$ be a smooth connected manifold. Associated with any Riemannian metric $\sigma$ on $M$ is a natural Lie subalgebroid $\mathfrak{g} \subset J^{1}(T M)$ which, according to [4], supports a canonical Cartan connection $\nabla$. The curvature of $\nabla$ vanishes on any simply-connected region $U$ precisely when the vector space of Killing fields on $U$ is of maximal possible dimension, and the Lie algebra of all such Killing fields can be concretely described. We now give a global analogue of this result.

By definition, $\mathfrak{g}$ is the subbundle of all one-jets $J_{m}^{1} V$ of vector fields $V$ on $M$ such that $\sigma$ has vanishing Lie derivative at the point $m \in M$. To describe the Cartan connection $\nabla$ explicitly, let $\nabla^{\mathrm{LC}}$ denote the Levi-Cevita connection, which we may regard as a splitting of the canonical exact sequence,

$$
0 \rightarrow T^{*} M \otimes T M \rightarrow J^{1}(T M) \rightarrow T M \rightarrow 0 .
$$

There is a corresponding exact sequence,

$$
0 \longrightarrow \mathfrak{h} \longrightarrow \mathfrak{g} \longrightarrow T M \longrightarrow 0,
$$

where $\mathfrak{h} \subset T^{*} M \otimes T M$ denotes the $\mathfrak{o}(n)$-bundle of all $\sigma$-skew-symmetric tangent space endomorphisms, which is $\nabla^{\mathrm{LC}}$-invariant. Because $\sigma$ is $\nabla^{\mathrm{LC}}$-invariant, the splitting subbundle of $J^{1}(T M)$ lies inside $\mathfrak{g}$ and we obtain an identification $\mathfrak{g} \cong T M \oplus \mathfrak{h}$. Under this identification, the Cartan connection $\nabla$ is given by

$$
\nabla_{U}(V \oplus \phi)=\left(\nabla_{U}^{\mathrm{LC}} V+\phi(U)\right) \oplus\left(\nabla_{U}^{\mathrm{LC}} \phi+\operatorname{curv} \nabla^{\mathrm{LC}}(U, V)\right) .
$$

With the help of the Bianchi identities for linear connections, one computes

$$
\operatorname{curv} \nabla\left(U_{1}, U_{2}\right)(V \oplus \phi)=0 \oplus\left(-\left(\nabla_{V}^{\mathrm{LC}} \operatorname{curv} \nabla^{\mathrm{LC}}+\phi \cdot \operatorname{curv} \nabla^{\mathrm{LC}}\right)\left(U_{1}, U_{2}\right)\right),
$$

implying implying that $\nabla$ is flat if and only if $\operatorname{curv} \nabla^{\mathrm{LC}}$ is both $\nabla^{\mathrm{LC}}$-invariant and $\mathfrak{h}$-invariant. Now $\mathfrak{h}$-invariance implies, by purely algebraic arguments, that curv $\nabla^{\mathrm{LC}}$ has only a scalar component:

$$
\operatorname{curv} \nabla^{\mathrm{LC}}\left(V_{1}, V_{2}\right)=s\left(\sigma\left(V_{2}\right) \otimes V_{1}-\sigma\left(V_{1}\right) \otimes V_{2}\right),
$$

for some function $s \in \mathbb{R}$, the scalar curvature; $\nabla^{\mathrm{LC}}$-invariance implies $s$ is a constant.

With a view to applying Proposition 6.5 in the flat case, we first define $\mathfrak{g}_{0}$ and $\mathfrak{h}_{0} \subset \mathfrak{g}_{0}$ as in Proposition 5.1, and, to obtain the bracket on $\mathfrak{g}_{0}$, compute the torsion, tor $\bar{\nabla}$, of the associated $\mathfrak{g}$-connection $\bar{\nabla}$ on $\mathfrak{g}$ (which is actually a $\mathfrak{g}$-representation). To this end, one requires a formula for the Lie bracket on $\mathfrak{g} \subset J^{1}(T M)$. Indeed, under the identification $\mathfrak{g} \cong T M \oplus \mathfrak{h}$, we have

$$
\left[V_{1} \oplus \phi_{1}, V_{2} \oplus \phi_{2}\right]_{\mathfrak{g}}=\left[V_{1}, V_{2}\right]_{T M} \oplus\left(\left[\phi_{1}, \phi_{2}\right]_{\mathfrak{h}}+\nabla_{V_{1}}^{\mathrm{LC}} \phi_{2}-\nabla_{V_{2}}^{\mathrm{LC}} \phi_{1}+\operatorname{curv} \nabla^{\mathrm{LC}}\left(V_{1}, V_{2}\right)\right),
$$

where $[\cdot, \cdot]_{\mathfrak{h}}$ is the bracket on the $\mathfrak{o}(n)$-bundle $\mathfrak{h}$. This formula is an instance of the general formula [4, Proposition 6.2(4)]. Supposing that $\nabla$ is flat, and hence (7.1) holds, one obtains with the help of (7.2),

$$
\text { tor } \bar{\nabla}\left(V_{1} \oplus \phi_{1}, V_{2} \oplus \phi_{2}\right)=\left(\phi_{1}\left(V_{2}\right)-\phi_{2}\left(V_{1}\right)\right) \oplus\left(\left[\phi_{1}, \phi_{2}\right]_{\mathfrak{h}}-s\left(\sigma\left(V_{2}\right) \otimes V_{1}+\sigma\left(V_{1}\right) \otimes V_{2}\right)\right) \text {. }
$$

Restricting tor $\bar{\nabla}$ to obtain the Lie bracket on $\mathfrak{g}_{0}=\left.\mathfrak{g}\right|_{m_{0}}$, we see from the formula that $\mathfrak{g}_{0}$ is the Lie algebra of $\mathfrak{o}(n) \times \mathbb{R}^{n}$ (semidirect product), $\mathfrak{o}(n+1)$, or $\mathfrak{o}(n, 1)$, according to whether $s=0, s>0$, or $s<0$, respectively. In each case $\mathfrak{h}_{0} \subset \mathfrak{g}_{0}$ is isomorphic to $\mathfrak{o}(n)$.

By definition, the metric $\sigma$ is $\mathfrak{g}$-invariant, so that completeness of $M$, as a metric space, implies completeness of the Cartan connection $\nabla$, by Proposition 6.3. In that case, Proposition 6.5 establishes the following: 
Proposition 7.2 (cf. Proposition 8.4.3 of [9]). If a connected Riemannian manifold $M$ is a complete metric space and has constant scalar curvature s. Then:

(1) The universal cover of $M$ is:

- $G_{0} / H_{0} \cong \mathbb{R}^{n}$, in the case $s=0$, where $G_{0}$ is the simply-connected Lie group with Lie algebra $\mathbb{R}^{n} \times \mathfrak{o}(n)$ (semidirect product);

- $G_{0} / H_{0} \cong S^{n}$, in the case $s>0$, where $G_{0}$ is the simply-connected Lie group with Lie algebra $\mathfrak{o}(n+1)$;

- $G_{0} / H_{0} \cong \mathbb{H}^{n}$, in case $s<0$, where $G_{0}$ is the simply-connected Lie group with Lie algebra $\mathfrak{o}(n, 1)$.

In every case $H_{0} \subset G_{0}$ is the connected subgroup with Lie algebra $\mathfrak{o}(n)$, realised as a subalgebra of $\mathfrak{g}_{0}$ in the usual way.

(2) The group of covering transformations $\Gamma \cong \pi_{1}(M)$ acts on $G_{0} / H_{0}$ by affine transformations.

\section{Acknowledgements}

The author acknowledges many helpful discussions with Ercüment Ortaçgil.

\section{References}

[1] Abadoğlu E., Ortaçgil E., Intrinsic characteristic classes of a local Lie group, Port. Math. 67 (2010), 453-483, arXiv:0912.0855.

[2] Alekseevsky D.V., Michor P.W., Differential geometry of $\mathfrak{g}$-manifolds, Differential Geom. Appl. 5 (1995), 371-403, math.DG/9309214.

[3] Blaom A.D., Geometric structures as deformed infinitesimal symmetries, Trans. Amer. Math. Soc. 358 (2006), 3651-3671, math.DG/0404313.

[4] Blaom A.D., Lie algebroids and Cartan's method of equivalence, Trans. Amer. Math. Soc. 364 (2012), 3071-3135, math.DG/0509071.

[5] Blaom A.D., A Lie theory of pseudogroups, in preparation.

[6] Bott R., Tu L.W., Differential forms in algebraic topology, Graduate Texts in Mathematics, Vol. 82, SpringerVerlag, New York, 1982.

[7] Crainic M., Fernandes R.L., Lectures on integrability of Lie brackets, in Lectures on Poisson Geometry, Geom. Topol. Monogr., Vol. 17, Geom. Topol. Publ., Coventry, 2011, 1-107, math.DG/0611259.

[8] Dazord P., Groupoïde d'holonomie et géométrie globale, C. R. Acad. Sci. Paris Sér. I Math. 324 (1997), $77-80$.

[9] do Carmo M.P., Riemannian geometry, Mathematics: Theory \& Applications, Birkhäuser Boston Inc., Boston, MA, 1992.

[10] Goldman W.M., Locally homogeneous geometric manifolds, in Proceedings of the International Congress of Mathematicians, Vol. II, Hindustan Book Agency, New Delhi, 2010, 717-744.

[11] Gunning R.C., Special coordinate coverings of Riemann surfaces, Math. Ann. 170 (1967), 67-86.

[12] Kamber F.W., Michor P.W., Completing Lie algebra actions to Lie group actions, Electron. Res. Announc. Amer. Math. Soc. 10 (2004), 1-10, math.DG/0310308.

[13] Kowalski O., On strictly locally homogeneous Riemannian manifolds, Differential Geom. Appl. 7 (1997), 131-137.

[14] Mackenzie K.C.H., General theory of Lie groupoids and Lie algebroids, London Mathematical Society Lecture Note Series, Vol. 213, Cambridge University Press, Cambridge, 2005.

[15] Palais R.S., A global formulation of the Lie theory of transformation groups, Mem. Amer. Math. Soc. 22 (1957), iii+123 pages.

[16] Sharpe R.W., Differential geometry. Cartan's generalization of Klein's Erlangen program, Graduate Texts in Mathematics, Vol. 166, Springer-Verlag, New York, 1997.

[17] Thurston W.P., Three-dimensional geometry and topology, Vol. 1, Princeton Mathematical Series, Vol. 35, Princeton University Press, Princeton, NJ, 1997. 